\section{SOME REMARKS ON CERTAIN PATHO- LOGICAL CONDITIONS OF THE OVARIES ASSOCIATED WITH MENORRHAGIA.}

BY C. J. BOND, F.R.C.S. ENG.,

SURGEON TO THE LEICESTER INFIRMARY.

Now that diseased appendages are removed at a much earlier stage of the morbid processes than formerly, and that oöphorectomy is probably a commoner operation than ovariotomy, much light has been thrown on certain early morbid states of the ovaries, some of which are associated with, if not the cause of, marked disturbance of uterine function. It is no doubt true that the Fallopian tube is so constantly included with the ovary in any operative procedure for the treatment or removal of the latter, that a corresponding association is apt to exist in the mind of the operator as to the functions of the two organs. No doubt pathological conditions of the tubes, especially those which produce cystic distension, are frequently found in cases of menorrhagia, but is it not most usual to find in these cases of cystic salpingitis corresponding inflammatory changes in or around the ovaries, including the latter within the diseased area? On the other hand, pathological conditions of the ovaries such as those to be mentioned later, of which menorrhagia is often a prominent symptom, are frequently found in connexion with perfectly normal conditions of the tubes, and it is only cases of such a kind where the tubes are healthy that $I$ propose to include in these remarks.

In looking through the notes of some fifty cases of removal of ovaries or appendages for various causes, which have come under my care during the last few years, there is a small group of eight cases in which small cystic ovaries were removed, in which the tubes were healthy, and in five of which the prominent symptom was menorrhagia of a pronounced type, being in three of them of a most violent kind-as violent and uncontrollable as is found in the worst cases of fibroids and immediately threatening life. In these five cases the ovaries were of the type described by Lawson Tait, Pozzi, and others as the "small cystic ovary." That is, although there was some considerable amount of ovarian tissue left, yet, in addition to a marked connective tissue proliferation or oöphoritis, the organs contained several, or numerous cysts varying in size from that of a nut to that of a walnut or larger; and a further point to which I would draw attention is the fact that these cysts contained dark blood-stained serum-there had been, in fact, extravasation of blood into the cystic cavities, and thus an indication is given of the amount of ovarian engorgement in these cases. In the remaining three cases the cysts contained clear flaid, the marked congestion was absent, and the symptoms lacked the pronounced one of excessive menorrhagia, although prominent menstrual disturbance of a painful and nervous kind was present. It has, of course, been known for some time that these small cystic ovaries are frequently associated with menorrhagia. This has been pointed out by Lawson Tait ${ }^{1}$ and by Pozzi ${ }^{2}$; but I doubt whether it is even yet sufficiently realised by medical practitioners, that persistent and violent menorrhagia, uncontrollable by curetting, may, and does, occur with a normal, or only slightly enlarged, uterus, and is due to ovarian disease alone. There is, I think, no doubt that the connexion between the sanguinolent character of the cyst contents and the excessive uterine hæmorrhage is more than a mere coincidence. The blood-stained fluid means severe ovarian engorgement, leading to extravasation, and it is probable that an engorged ovary provides a more powerful stimulus to the menstrual process than a healthy one.

In spite of the absence of direct evidence of the part played by the various organs in the menstrual process, one may, I think, usefully adopt as a working hypothesis a modification of the scheme of the menstrual nervous mechanism suggested by Martin ${ }^{3}$ and regard it as consisting of a spinal centre in the lumbar region not far from the centre for parturition. This receives afierent impressions from the ovary by way of the ovarian plexus, from the uterus, and from distant parts - such as the breast and the brain-the

1 Disease of the Ovaries, fourth edition, 1884. 2 Treatise on Gynæcology.

3 Provincial Medical Journal, December, 1893. efferent channels from this centre pass to the uterus along the uterine nerves in the broad ligament. One may, in fact, regard the ovarian tissue as the peripheral end organ of one of the afferent channels.

As regards the nature of the stimulus, or the manner in which the orary affects the uterus, it is, I think, clear from much recorded evidence of the condition of the ovaries re moved during menstruation, that ovulation and menstruation are not necessarily synchronous-the former precedes the onset of menstruation by many years, and may go on after the latter has ceased. The ovary appears rather to influence the aterns by means of a continuous and steady nerrous impulse, tending to stimulate the development and changes of the mucons membrane in so far as these are concerned in providing a nidus for the ovam, and acts in the way of causing the renewal of decidual development after each parturition or menstrual period, rather than in bringing about its destruction. by menstruation. The ovary acts, as a whole, upon the uterus in the same way that a single ovum within the uterus acts upon it. If the ovarian stimulus be thus continuous and constructive in its character, one must look elsewhere for an explanation of the destructive and periodic phenomena which are so characteristic of menstruation. In speculating for a moment as to the steps by which this apparently useless function has been evolved in organic life, it is a sug. gestive fact, that, owing perhaps to a more regular and even food-supply, and protection from climatic influences which affect the young progeny, an alteration in ovarian fertility has occurred in the human species, so that one or a few mature ova are produced at frequent intervals during fertile life, instead of a larger number of ova at longer intervals. Accompanying this change in ovarian activity there has also been brought about a corresponding change in uterine activity, which I would explain as follows. It is probable, for several reasons, that the natural life of the epithelial lining of that portion of the oviduct specially differentiated for the reception of the ovum is, or rather must be, a comparatively short one-that is, an impregnated ovum, if it is to become attached and grow, it requires a young membrane, or one from which the older epithelial cells have been shed, in which it can become imbedded; and it may be that the closer and more bighly developed the utero-fœtal connexionin other words, the placenta-the more is this a matter of importance. In the case of the lower animals, in which orvation, or rather the possibility of impregnation, occurs at at relatively long intervals, this change in the uterine mucous surface is accomplished by a gradual absorption of the older cell elements and regeneration of new ones by atrophy and lymphatic absorption, without any visible external escape of blood or throwing off of epithelium. The higher apes appear to stand in an intermediate position, and the in creased cellular infiltration of the membrane at the cestrual periods of these animals, as described by Bland Sutton, supports this view. In fact, menstruation is a more rapid way of getting rid of an epithelial surface no longer fit to serve as a nidus for the growing ovum, the increased rapidity being necessitated by the more frequent ovulation and opportunities of impregnation, or the constant condition of receptivity characteristic of the human female. Löwenthal, taking a view somewhat like this, attributes the growth and shedding of the epithelial lining in the human uterus, to the influence of the unimpregnated ovum in contact with it, and he regards the life of the two as coterminous ; in this I think he errs, as in such a case the advent of a second ovum from the same, or the other ovary, before the death of the first, would keep alive the epithelial surface, and so there would frequently be arrest of menstruation without pregnancy, whereas this does not occur in physiological health. Thus, though the occurrence of menstruation may be quite independent in point of time with the maturation and rupture of the Graafian follicle, yet the process is a highly specialised form of the nidation changes which occur in the oviduct in a cyclical manner, and is dependent on and subservient to the ovarian function. Thus menstruation is really analogous to parturition, while during pregnancy the strong stimulus of the growing, impregnated ovum prevents the degeneration and shedding of the epithelial surface on which it is implanted, and prolongs its life for a period of time which has a definite relation to the menstrual cycle.

In the case of those lower aniraals, chiefly smaller ones, in which, relatively to their size, food supply is abundant and climatic influence is modified by habits-such as rats, mice, and rabbits, in which ovulation and impregnation, or the opportunities for it, occur at frequent intervals-it would 
at first sight appear as if some process of menstruation would be necessary in their case, as in the human species, to bring about the more rapid removal and renewal of the membrane necessitated by the more frequent ovulation or cycle of nidation; but it must be remembered that in the state of nature, the opportunity of impregnation is practically coexistent and synchronous with ovulation, and so parturition rapidly accomplishes for such animals at the end of the cycle, that which menstruation effects in unimpregnated women, or the slower process of absorption in the animals which ovulate at longer intervals.

To sum up, it appears that ovulation and nidation, of which menstruation is a specialised form, evolved to meet certain altered conditions of life, are both dependent, as regards their frequency and periodicity, on the periods of receptivity of the animal to sexual congress or the cestrus, and that these latter are again determined by climatic conditions and food-supply, operating by means of natural selection on the production and rearing of progeny. Further, it appears that, ovulation and nidation, or menstruation, having thus been associated together so long in one nervous mechanism, any interference with the one, as by removal of the ovaries, inhibits or interferes with the other. Be this as it may, by the supposition of some such nerve mechanism, it is more easy to understand how the menstrual process itself may be inhibited, or altered by any interference with the afferent channel, consisting of ovary and ovarian nerves, or with the centre in the spinal cord, or with the efferent nerves passing to the uterus by way of the broad ligament; thus complete removal of both ovaries removes the whole of the afferent end organ, while removal of the tubes, and other operations on the broad ligament, are effective only in so far as they obliterate the afferent or efferent channels, or in some cases interfere with the vascular supply. By some such hypothesis, too, one may interpret the fact that, in the worst cases of menorrhagia due to orarian causes, menstruation is often painless, while in other cases in which menorrhagia is not a prominent symptom the pain and other nervous manifestations are very marked, as though the afferent impulses from the ovary in the first case were reflected downwards from the centre to the aterns in a trophic and secretory stream, and in the latter case upwards to the brain along the sensory channels. This at present unfortunately lacks the support or the contradiction of experimental investigation; some light on the nervous mechanism might, however, perhaps be thrown by the clinical observation of spinal injury in the female.

But to return to the small cystic ovary. The differences between this form and the ovary affected with the large cysts of the ovarian cystoma capable of indefinite growth are very marked clinically, since the latter form has very little influence on the menstrual process, and from the researches of Gallard it appears to as frequently cause a diminished flow as an increased one. Secondly, pathologically, the researches of many observers-Waldeyer, Doran, and others-seem to show that the ordinary ovarian cystoma originates in an epithelial overgrowth of a glandular type, with minor differences peculiar to the various forms of cyst. On the other hand, the early changes in the small cystic variety have not been fully worked out ; it has, however, no tendency to pass into the common form of cystoma, and from the examination of numbers of sections of such ovaries removed by myself it appears, I think, certain that the early change is one of epithelial degeneration rather than growth, with effusion or extravasation into such degenerated follicles, which become recognised as cysts. Such sections show a more or less irregular cavity filled with the coagulated serous or bloody material, and this merges directly into the fibro-vascular tanic without the intervention of an epithelial layer. Sometimes some epithelium can be found in an irregular and degenerated condition, and the surrounding ovarian stroma shows all degrees of connective tissue overgrowth, the result of oöphoritis, and the surface of the organ often has a toughened, leathery, dull character from the same cause. It is, I think, clear that these cysts originate in Graafian follicles, which perhaps in a few cases undergo a pathological change after rupture. In some rarer cases cystic change may occur in a corpus luteum itself, as mentioned by Pozzi, or more often in those cysts which, more deeply placed in the substance of the ovary, fail to reach the surface, perhaps owing to the increased fibrous character of the ovarian tissue, and which undergo the cystic degeneration without even rupturing at all. The main feature of the change is that of disordered involution. Extravasation occurs from the surrounding vessels into the cavity, and, lacking the safety valve of the normal rupture at the time of shedding the ova, the follicles tend to enlarge mechanically up to a certain limited size, as opposed to the unlimited enlargement by epithelial growth, and secretion, of the larger cystomata. The real difference between the condition of the small cystic ovary, which increases the menstrual flow, and the ovary in ordinary cystoma, which does not affect it, is one of vascularity and function; in the former case there is vascular engorgement and cysts which in the early stages are functionally active ; in the latter there is less vascularity and functionless cysts as far as ovulation is concerned, and the irritated or small cystic ovary, by transmitting a stronger stimulus of nidation, causes a more vascular uterus, with a corresponding increase of hæmorrhage when the membrane is shed and extravasation occurs. It is, in fact, as if the exaggerated change which occurs in a ruptured follicle with pregnancy, which is dignified by the name of "corpus luteum," occurred in an unruptured follicle in an exaggerated degree, as if the ovarian stimulus were one of constant pregnancy or overgrowth of decidual membrane, while the uterus lacks the inhibitory influence of the encapsuled ovum to prevent its breaking down. A true view of the pathology of such ovarian disease would, I think, regard it as an advanced condition of the state which is found in many ovaries, even in those of young unmarried women; such ovaries are called "normal," and they are so in the sense that they cause no symptoms or disorders of menstruation, but the condition is really pathological, in the sense that the normal evolution and involation of the follicle are not carried out, and it is an interesting point as to the generality and existence of this faulty physiology in the various grades of the animal kingdom. It is known that mares are especially liable to the change. The fact that all stages can be found, between the so-called normal ovary and the small cystic ovary which produces such marked symptoms, is a strong argument in farour of the view that the latter condition is the terminal stage in a pathological series.

Clinically, these small cystic ovaries not only cause increased menstruation, but in some cases increase in size of the body of the uterus - not necessarily increase of area of cavity, but increase in the thickness of the uterine wall ; the organ is in these cases big, sodden, and heavy, and it is possible that some cases, which show a great tendency to flooding: after miscarriages, are caused by a want of uterine vigour and tone, being thus indirectly due to diseased ovaries, and that, in the cases of nnexplained recurring tendency to early abortion, the real source of the disease is the excessive ovarian stimulus to the spinal centres, overcoming the inhibitory influence of the growing ovum, and thus producing a menstruation or early parturition. And if the lesson of the use of the pessary in uterine displacement be fairly studied it will, I think, be found to be more and more the case that so-called displacements of a normal uterus cause but few symptoms when the appendages are healthy, but that where uterine displacements do cause symptoms, those symptoms are either due to, or exaggerated by, the abnormal condition of the ovaries or tubes upon which the displacement acts. These small cystic ovaries also in some cases prolong menstruation long after the normal period of uterine involution. I have seen one case of somewhat excessive menstruation at the age of fifty-eight in which the ovaries were cystic. In cases of uterine fibroids the ovaries are frequently cystic, as has been mentioned by Lawson Tait, and I have several times seen the cyst contents sanguinolent in this affection. How far the ovarian change is a secondary one, or how far it has a primary influence in producing not only the menorrhagia, but also the overgrowth in the uterus, it is not possible to say at present. Many cases, however, occur in which the ovaries are not cystic, and on the other hand the engorgement of the uterus may extend to the ovary and produce by increased congestion the pathological change in the follicles; this is rendered probable by the fact that an enlarging uterus in the case of pregnancy exercises some influence on the ovaries, as is shown by the peculiarities of the formation of the corpus luteum.

With regard to the treatment of these cases, a true appreciation of their pathology, and a recognition of the ovarian origin of the uterine symptoms, will lead to a more rational and therefore a more successful, treatment. The futility of curetting as a means of permanent cure and the uselessness of all forms of merely intra-uterine meciuation or mechanical treatment, except as palliative means, will be understood: the pelvis will be more frequently explored from above, and 
the ovaries themselves will be treated. While it is right to have certain rules clearly defined in one's own mind as guides in coming to a decision, in each individual case, as to the desirability, or otherwise, of removal of the ovaries, the subject is still too much the battle-ground of gynæcological opinion to allow the expression of any dogmatic statement. Personally, I should be inclined to say that where, in the absence of other causes, there is reason to believe that the cause of the symptoms lies in the ovaries, and that further the symptoms are of sufficient intensity to either endanger life or to lead to chronic invalidism, a case is made out for abdominal section and exploration; that, further, having the appendages within sight, or touch, or both, the guide should be as follows: Any gross disease of tubes or markedly pathological state of ovaries of course decides the point at once, whereas in the case of lesser degrees of pathological change, where the oöphoritis is not very marked or the cysts are few and small, the fact already mentioned-namely, that such a condition is in reality one stage in a pathological series from normality to marked diseaseshould be borne in mind, and the decision should be taken, not so much on the amount of disease detectable by sight or touch in the ovaries, as on the intensity of the symptoms, and especially the clearness of the evidence which connects them with an ovarian origin, and the absence of other evidence, especially of central nervous system origin. Hitherto I have spoken only of total removal of the diseased organs, but already there has been suggested and put into practicenotably by Pozzi and others - a conservative treatment of the appendages; it consists in the freeing and replacement of adherent and displaced organs and in the evacuation of small cysts, with the destruction of their lining membrane by cauterisation. As far as conservative treatment is concerned with the liberation of adherent organs and the replacement of displaced ones, there can, I think, be no doubt that the principle is sound and the future promising ; but, when dealing with such morbid conditions as the cystic change mentioned above, it is necessary to speak with caution, for if the functional origin of these cysts is borne in mind it is, I think, justifiable to express a doubt whether an ovary which has commenced to functionate in this abnormal manner will not continue to do so even after the evacuation and cauterisation of any cysts already formed.

I have so far spoken only of this special and peculiar change in the ovary, characterised by a fibrous overgrowth due to oöphoritis, and the presence of small cysts as a cause of menorrhagia, and for this condition radical treatment is required. The experience of every one will of course suggest the association of menorrhagia of varying degrees of severity with less advanced pathological changes-for instance, a common cause of minor degrees of menorrhagia is a prolapsed, and therefore a congested, ovary, with its periodic hypercongestion at the monthly periods; there are also the various causes which produce congestion of the ovary without displacement.

Leicester.

\section{FOUR CASES OF EXTRA-UTERINE GESTA- TION SUCCESSFULLY TREATED BY ABDOMINAL SECTION. ${ }^{1}$}

BY F. F. SCHACHT, M.D. CANTAB., PHYSiCian to OUT.PATIENTS AT CHELSEA hOSPITAL For WOMEN.

IT so happened that between the months of May and November, 1893, four cases of ectopic gestation (all within the first four months of pregnancy) came under my notice at the Chelsea Hospital for Women. The first case was sent to me by Mr. Farr of Kensington, who had already diagnosed the condition, while the other three cases were, or had been, under my care as out-patients, and were sent into the hospital by me. The second and fourth cases went into Dr. Travers' beds and were successfully operated on by him ; while the third case, like the first, was under my charge. Individually they appear to mo to present many points of interest, all of which it is impossible to touch upon here ; while taken together they illustrate very clearly some of the difficulties of diagnosis and pathological possibilities

1 A paper read before the West London Medico.Chirurgical Society on Jan. 5th, 1891, when specimens were also shown. associated with this special condition. The notes of each case are abbreviated as much as possible in regard to operation and after results, partly because the cases did well, and partly because I feel that the main interest must lie in the previous history, condition on admission to hospital, and diagnosis.

CASE 1.-A woman thirty years of age; she had been married eight years, and was the mother of two children, the last child being born four years ago; she had had no mis. carriage. The catamenia were regular till fourteen weeks previously. There was a slight show one week since for a night and day; clot and something fleshy were passed. Morning sickness had been present, but there was no fulness or pain in the mammæ. Seventeen days ago (May 2nd) the patient felt sudden pain while lying in bed, with faintness and sickness. The pain, which was like that at the menstrual period, was felt in the right lower abdomen, where for three months previously she had been liable to intermittent twisting, nauseating pain, irrespective of the position of the body. She had suffered from chronic pain in the right inguinal region of quite a different character during the last two years. She had been laid up since the onset of the pain seventeen days previously. That attack lasted two hours. The next day (the 3rd) similar pain was again experienced for about one hour. On the 17th, in the morning, a third attack of pain, this time more severe, was experienced. On this occasion she fainted. Mr. James Farr was called in and diagnosed ruptured tubal gestation, with which opinion his brother (Mr. Farr) agreed.

On admission to the Chelsea Hospital for Women the patient looked very ill and blanched, with that drawn expression of the features so suggestive of abdominal trouble and pain. The temperature was $97.8^{\circ} \mathrm{F}$., the pulse 104, and the respiration 28. On examination the mammæ were found to be somewhat enlarged; there was some darkening of the areolæ; drops of watery secretion could with difficulty be obtained; there was slight general abdominal fulness; tender. ness was not general, but across the lower abdomen, especially in the right inguinal region, where on light palpation was felt a roundish mass-not very hard-rising to about two inches below the umbilicus, and extending from the central line almost to the ileum. Over this region there was dulness on percussion, whereas on the left side the pereassion note was clear. On examination per vaginam the cervix was found to be fairly hard to the left of the median line. The uterus was apparently pushed over to the left by a mass which occupied the right fornix. This mass was continuons with the mass felt per hypogastrium; it gave the idea of a rounded elongated tense semi-solid mass. In Douglas' pouch there was felt a rather harder rounded mass, only a slight depression separating it from the former. To the right of the cervix, as the finger passed on to the mass, there was felt a vessel distinctly pulsating. Nothing further was elncidated per rectum. A consultation was at once held, and an immediate operation was decided upon.

After ether was administered the usual abdominal incision was made. On opening the peritoneal cavity blood welled up. The mass previously felt proved clearly to be a much distended right tube, pushing a somewhat enlarged uterus over to the left. It was drawn up gently, a bleeding point was temporarily con. trolled, a double silk ligature was tied close up to the uterus, and the mass was removed. On mopping out Douglas' pouch with a sponge a large blood clot was turned out in which the foetus was enveloped. About eight ounces of fluid blood, besides many clots, were removed during the operation. The abdominal cavity was freely washed out with hot water, all clots removed, a Keith's glass drainage-tube inserted, and the wound brought together. The progress of the case was for the first fourteen days satisfactory. The drainage-tube was removed at the end of forty-eight hours, two ounces of redcoloured fluid only being drawn off, notwithstanding that a considerable quantity of water had been left in at the operation. The only cause for any enxiety lay in the pulse, which varied from 130 to 140 during the forty-eight hours after the operation. The patient was consequently fed with nutrient enemata and brandy every four hours. The general condition slowly improved; but though the pulse fell to 104 on the fifth day it was still over 100 on the tenth day. No doubt there was a distinct nervous element in it. The vascular condition at this time existing probably accounted for the "white swelling" which began to manifest itself in the left leg on the sixteenth day. This condition, after being acute for three days, gradually subsided, and the patient was sent to the convalescent home six weeks after the operation. 